\title{
Add-On Treatment with Teneligliptin Ameliorates Glucose Fluctuations and Improves Glycemic Control Index in Japanese Patients with Type 2 Diabetes on Insulin Therapy
}

\author{
Seiichi Tanaka, MD, Kunihiro Suzuki, MD, PhD, Chie Aoki, MD, PhD, Mai Niitani, MD, \\ Kanako Kato, MD, Takanori Tomotsune, MD, and Yoshimasa Aso, MD, PhD
}

\begin{abstract}
Background: This study investigated whether teneligliptin, a novel dipeptidyl peptidase-4 inhibitor, ameliorated glucose fluctuations in hospitalized Japanese patients with type 2 diabetes receiving insulin therapy, with or without other antidiabetes drugs, and using continuous glucose monitoring (CGM).

Patients and Methods: Twenty-six patients with type 2 diabetes were admitted for glycemic control. After admission, patients continued to be treated with optimal dietary therapy plus insulin therapy, with or without other antidiabetes drugs, until they achieved stable glycemic control. CGM measurements were made for 7 consecutive days. On Days 1-3, patients received insulin with or without other antidiabetes drugs, and on Days 4-7, teneligliptin $20 \mathrm{mg}$ once daily at breakfast was added to ongoing therapy. Doses of insulin were fixed during the study. Levels of serum glycated albumin (GA), 1,5-anhydro-D-glucitol (1,5-AG), and high-sensitivity C-reactive protein (hsCRP) were measured.

Results: Add-on treatment with teneligliptin led to significant improvements in 24-h mean glucose levels, the proportion of time in normoglycemia, mean amplitude of glycemic excursions, and total area under the curve within $2 \mathrm{~h}$ after each meal. The proportion of time in hypoglycemia and hsCRP levels did not increase significantly compared with before teneligliptin. Values of 1,5-AG and GA were significantly improved by treatment with teneligliptin.

Conclusions: Addition of teneligliptin to insulin therapy led to a significant improvement in diurnal glycemic control and significant reductions in glucose fluctuations in 24-h periods without increasing hypoglycemia in Japanese patients with type 2 diabetes on insulin therapy, with or without other antidiabetes agents.
\end{abstract}

\section{Introduction}

G LUCAGON-LIKE PEPTIDE-1 IS AN INCRETIN hormone that plays an important role in regulating postprandial blood glucose levels in a glucose-dependent manner. ${ }^{1,2}$ However, its rapid degradation by dipeptidyl peptidase-4 (DPP-4) means that native glucagon-like peptide-1 is not suitable for clinical use. ${ }^{3-5}$ Clinically, inhibition of DPP-4 can help control fasting and postprandial glucose levels by increasing plasma concentrations of active glucagon-like peptide-1. ${ }^{5,6}$ Therefore, DPP-4 inhibitors, incretin-based agents, are a promising new class of antidiabetes drug that can improve glucose fluctuations in patients with type 2 diabetes. $^{7}$
Teneligliptin, a novel DPP-4 inhibitor, is substantially metabolized in the liver, and its concentration in the serum is not affected even by severe renal impairment. ${ }^{8}$ This suggests that teneligliptin can be administered to patients with renal impairment due to diabetic nephropathy without the need for dose adjustments. ${ }^{8}$ This property, which differentiates teneligliptin from other DPP-4 inhibitors, enables us to prescribe this DPP-4 inhibitor to patients with type 2 diabetes who have overt diabetic nephropathy. Moreover, a recent study has shown that teneligliptin has a high potency of inhibiting DPP-4 activity compared with other DPP-4 inhibitors, ${ }^{9}$ including sitagliptin, ${ }^{10}$ saxagliptin, ${ }^{11}$ alogliptin, ${ }^{12}$ linagliptin,${ }^{13}$ and vildagliptin. ${ }^{14}$

Department of Endocrinology and Metabolism, Dokkyo Medical University, Tochigi, Japan. 
Insulin therapy is the most potent antihyperglycemic agent available for inadequately controlled patients with type 2 diabetes. ${ }^{15}$ Combination therapy of insulin and conventional oral antidiabetes drugs has been reported to be more effective than insulin alone. ${ }^{15-17}$ Advantages of combination therapy with insulin and a DPP-4 inhibitor, such as sitagliptin, include not only an improvement in glycemic control, but also prevention of weight gain, resulting in alleviation of the psychological stress that often occurs when insulin therapy is started. ${ }^{7,15}$ The use of teneligliptin as add-on therapy to other antidiabetes drugs was approved in December 2013 in Japan. So far, no reports have shown the efficacy of combination therapy of teneligliptin and insulin in patients with type 2 diabetes. Accordingly, in the present study, we investigated the effects of adding teneligliptin to ongoing insulin therapy, with and without other oral antidiabetes drugs, on 24-h glucose fluctuations in hospitalized patients using continuing glucose monitoring (CGM). The present study is the first report to demonstrate the efficacy of teneligliptin on glucose fluctuations validated by CGM in patients with type 2 diabetes.

\section{Patients and Methods}

\section{Subjects}

All patients had been admitted to Dokkyo Medical University Hospital (Tochigi, Japan) for glycemic control and during their hospitalization had achieved stable glycemic control with insulin monotherapy or insulin and other oral antidiabetes drugs (non-DPP-4 inhibitors). Inclusion criteria were (1) age $\geq 20$ years, (2) hemoglobin A1c level on admission of $\geq 7.5 \%$, (3) treatment with insulin therapy, including once-daily basal insulin with or without oral antidiabetes drugs, twice-daily premixed insulin therapy, or multiple daily insulin therapy, for at least 2 weeks, and (4) daily insulin dose of $\leq 60$ units. Exclusion criteria were (1) type 1 diabetes, (2) severe complications of diabetes, (3) severe renal and liver dysfunction, (4) severe infections, (5) pregnant or nursing women and those who might be pregnant, (6) alcoholism, and (7) any patients whom the investigators judged to be inappropriate for this study.

This study was approved by the Ethics Committee of Dokkyo Medical University. All subjects were given an explanation of the details of this clinical study and provided written informed consent. This study was designed in accordance with the principles stated in the Declaration of Helsinki.

\section{Study design}

This was a prospective, nonblinded, pilot study in 26 patients. After admission, all patients achieved stable glycemic control by insulin alone or insulin with other oral antidiabetes drugs, in addition to optimal dietary therapy. During the last week of hospitalization, glycemic levels were monitored for 7 consecutive days using CGM (ipro ${ }^{\circledR} 2$; Medtronic MiniMed Inc., Northridge, CA). During the study period, the dose of insulin was fixed, and $20 \mathrm{mg}$ of teneligliptin once daily after breakfast was added to ongoing therapy on Day 4 after the start of CGM measurements. As indexes of 24-h glucose fluctuations, we evaluated 24-h mean (SD) glucose levels, mean amplitude of glycemic excursions, rates of proportions of appropriate glucose levels (70-140 mg/dL), hyperglycemia ( $>140 \mathrm{mg} / \mathrm{dL})$, and hypoglycemia ( $<70 \mathrm{mg} / \mathrm{dL})$, total area under the curve (AUC) within $2 \mathrm{~h}$ after each meal, and postprandial glucose levels within $2 \mathrm{~h}$ after each meals using 24-h glycemic data from CGM.

Levels of serum glycated albumin (GA), 1,5-anhydro-Dglucitol (1,5-AG), and high-sensitivity C-reactive protein (hsCRP) were measured on Day 0 (the day that CGM was started), on Day 4 (the day that teneligliptin was added), and on Day 8 (the end of the study).

All patients carried a pedometer to meet the target of taking 5,000 steps a day under the guideline of medical staff during admission.

\section{Statistical analysis}

All statistical analyses were performed using GraphPad Prism $^{\circledR}$ version 6 for the Mac (GraphPad Software, San Diego, CA). Data are presented as mean \pm SD values. Differences between times points were assessed using the Wilcoxon signed rank test. A value of $P<0.05$ was considered significant.

\section{Results}

Demographic and clinical characteristics of the 26 subjects (13 men and 13 women; mean age, $60.8 \pm 15.7$ years) at study initiation are shown in Table 1 . The most common insulin regimen used was multiple daily injection therapy (13 patients [50.0\%]), and the most common antidiabetes drug was metformin (eight patients [30.7\%]). Variations in 24-h blood glucose levels measured by CGM during add-on treatment with $20 \mathrm{mg}$ of teneligliptin are shown in Figure 1. Add-on treatment with $20 \mathrm{mg}$ of teneligliptin significantly decreased both fasting and postprandial glucose levels on Days 5-7.

The indexes of glucose fluctuations derived from CGM are shown in Table 2. The mean 24-h blood glucose level was significantly decreased after treatment with teneligliptin $(P<0.001)$. Treatment with teneligliptin significantly increased the proportion of time in the normal range of glycemia $(P<0.001)$ and decreased the proportion of time in

Table 1. Baseline Characteristics of Study Subjects

\begin{tabular}{lc}
\hline Characteristic & Value \\
\hline Age (years) & $60.8 \pm 15.7$ \\
Sex $(\mathrm{M} / \mathrm{F})$ & $13 / 13$ \\
Body mass index $\left(\mathrm{kg} / \mathrm{m}^{2}\right)$ & $25.3 \pm 5.2$ \\
HbA1c $(\%[\mathrm{NGSP}]$ at admission) & $10.8 \pm 2.6$ \\
Urinary C-peptide $(\mu \mathrm{g} /$ day) & $43.9 \pm 29.1$ \\
Duration of diabetes (years) & $11.5 \pm 12.8$ \\
Insulin dose $(\mathrm{U} /$ day) & $22.5 \pm 16.0$ \\
Insulin therapy $[n(\%)]$ & \\
$\quad$ Twice & $4(15.4)$ \\
$\quad$ Multiple & $13(50.0)$ \\
$\quad$ Basal & $9(34.6)$ \\
Oral antidiabetes drug $[n(\%)]$ & \\
$\quad \alpha$-Glucosidase inhibitor & $5(19.2)$ \\
$\quad$ Biguanide & $8(30.7)$ \\
$\quad$ Glinide & $4(15.3)$ \\
$\quad$ None & $9(34.6)$ \\
\hline
\end{tabular}

Data are mean \pm SD values.

F, female; HbA1c, hemoglobin A1c; M, male; NGSP, National Glycohemoglobin Standardization Program, 


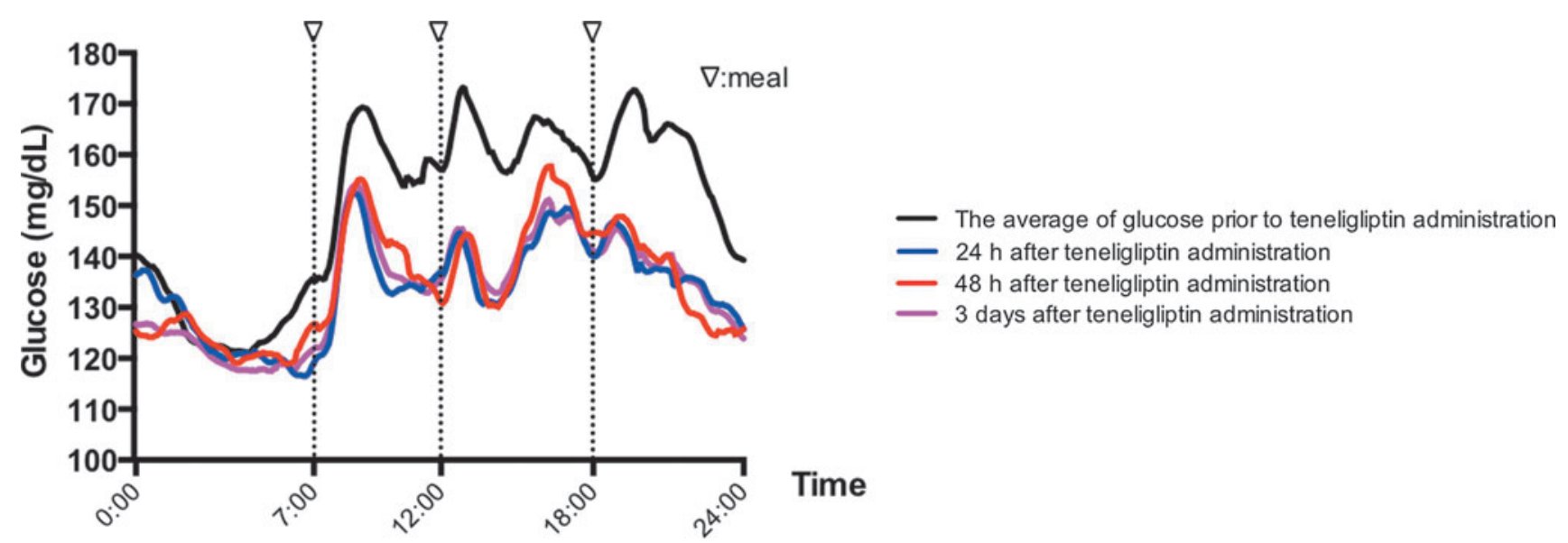

FIG. 1. The 24-h glucose profiles before and after administration of teneligliptin in hospitalized Japanese patients with type 2 diabetes receiving insulin therapy, with or without other antidiabetes drugs.

hyperglycemia $(P<0.001)$. There was no significant difference in the proportion of time in hypoglycemia before and after treatment with teneligliptin. The SD of 24-h glucose levels was lower in patients after treatment with teneligliptin than before treatment with teneligliptin $(P<0.001)$. The mean amplitude of glycemic excursions was significantly lower in

Table 2. Glucose Variability Parameters Before And After Treatment with Teneligliptin $(N=26)$

\begin{tabular}{lcc}
\hline & \multicolumn{2}{c}{ Insulin } \\
\cline { 2 - 3 } & $\begin{array}{c}\text { Before } \\
\text { tenegliptin } \\
\text { treatment }\end{array}$ & $\begin{array}{c}\text { After } \\
\text { tenegliptin } \\
\text { treatment }\end{array}$ \\
\hline Mean glucose level (mg/dL) & $148.8 \pm 25.7$ & $131.3 \pm 17.0^{\mathrm{b}}$ \\
0:00-7:00 h & $126.0 \pm 7.5$ & $119.0 \pm 4.2^{\mathrm{b}}$ \\
7:00-24:00 $\mathrm{h}$ & $159.1 \pm 9.8$ & $138.0 \pm 9.1^{\mathrm{b}}$ \\
Postprandial glucose & & \\
level (mg/dL) within & & \\
2 h after meal & & \\
Breakfast & $152.8 \pm 9.0$ & $140.0 \pm 10.9^{\mathrm{b}}$ \\
Supper & $164.8 \pm 2.7$ & $135.4 \pm 4.9^{\mathrm{b}}$ \\
Dinner & $165.8 \pm 5.3$ & $142.6 \pm 5.1^{\mathrm{b}}$ \\
SD over 24h (mg/dL) & $32.0 \pm 16.2$ & $26.9 \pm 10.9^{\mathrm{b}}$ \\
Proportion (\%) of time in & & \\
Hypoglycemia (<70 mg/dL) & $1.0 \pm 2.4$ & $1.6 \pm 2.6$ \\
Appropriate glucose & $38.3 \pm 24.5$ & $59.0 \pm 20.3^{\mathrm{b}}$ \\
level (70-140 mg/dL) & & \\
Hyperglycemia & $59.8 \pm 24.7$ & $37.6 \pm 22.4^{\mathrm{b}}$ \\
(>140 mg/dL) & & \\
MAGE (mg/dL) & $90.1 \pm 46.7$ & $85.5 \pm 34.3^{\mathrm{a}}$ \\
Total AUC (mg/dL/h) & & \\
for glycemic variability & & \\
within 2 h of each meal & $31.9 \pm 5.8$ & $29.0 \pm 5.3^{\mathrm{b}}$ \\
Breakfast & $33.4 \pm 8.4$ & $27.7 \pm 5.7^{\mathrm{b}}$ \\
Supper & & $28.4 \pm 5.1^{\mathrm{b}}$ \\
Dinner & & \\
\hline
\end{tabular}

Data are mean $\pm \mathrm{SD}$ values.

${ }^{\mathrm{a}} P<0.05,{ }^{\mathrm{b}} P<0.001$ versus before teneligliptin administration.

AUC, area under the curve; MAGE, mean amplitude of glycemic excursions. patients after treatment with teneligliptin than before treatment with teneligliptin $(P<0.05)$. In addition, treatment with teneligliptin significantly decreased postprandial glucose levels and total AUC for glycemic variability within $2 \mathrm{~h}$ after each meal.

Changes in indexes of blood glucose control and hsCRP occurred within a short period after teneligliptin administration. Serum GA levels decreased significantly after treatment with teneligliptin (Fig. 2A), whereas serum 1,5-AG levels increased significantly (Fig. 2B). There were no significant changes in serum hsCRP levels after treatment with teneligliptin (Fig. 2C).

Teneligliptin also significantly improved levels of both hemoglobin A1c $(10.8 \pm 2.6 \%$ at admission to $7.6 \pm 1.6 \%)$ and GA $(23.5 \pm 6.1 \%$ before treatment with teneligliptin to $19.8 \pm 3.1 \%$ after) at 6 months after the initiation of administration.

\section{Discussion}

We investigated the effects of a novel DPP-4 inhibitor, teneligliptin ( $20 \mathrm{mg}$ once daily after breakfast), on 24-h blood glucose levels in patients with type 2 diabetes receiving insulin therapy, with or without other antidiabetes drugs. Administration of teneligliptin provided a significant improvement in 24-h blood glucose profile, as well as decreases in SD of blood glucose levels over a 24-h period and mean amplitude of glycemic excursions, which are indexes of glucose fluctuations, without increasing hypoglycemia.

Some authors have reported that add-on treatment with DPP-4 inhibitors significantly improved glycemic control without increasing hypoglycemia in patients with type 2 diabetes on insulin therapy. ${ }^{15,18}$ In previous reports, the beneficial effect of sitagliptin represented mainly a postprandial glucoselowering effect, ${ }^{19,20}$ whereas some authors reported that the addition of sitagliptin to long-acting insulin or biphasic insulin therapy significantly decreased not only postprandial glucose levels but also preprandial glucose levels. ${ }^{18,21}$

Our results with teneligliptin were in agreement with these reports; however, teneligliptin also significantly decreased both pre- and postprandial glucose levels in a short period. The present study is the first report examining the effect of 
A

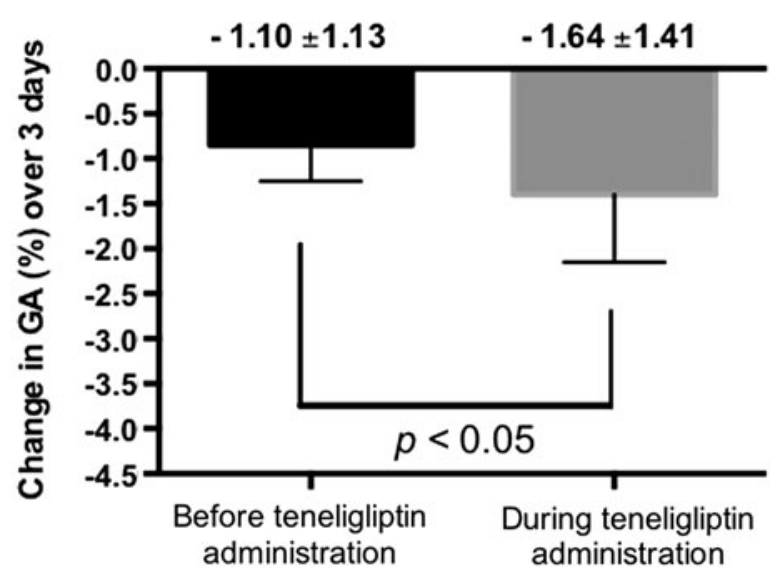

B

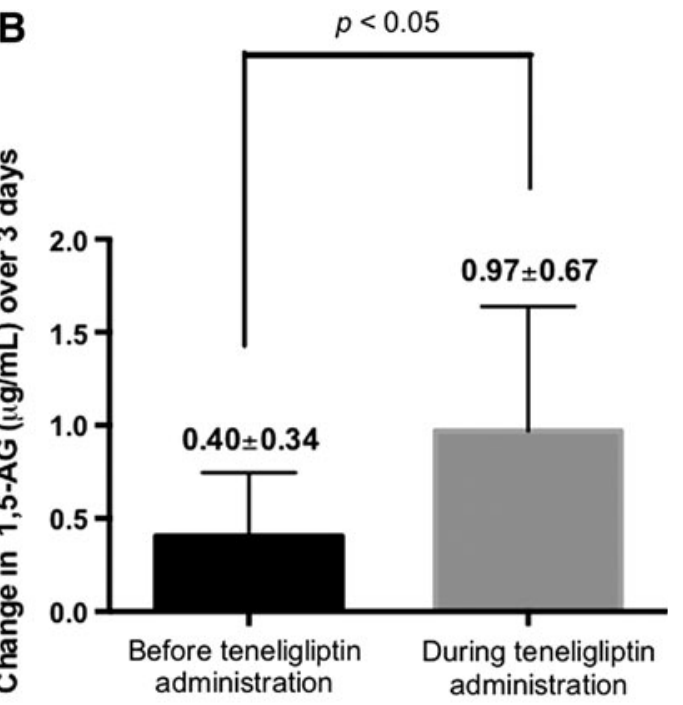

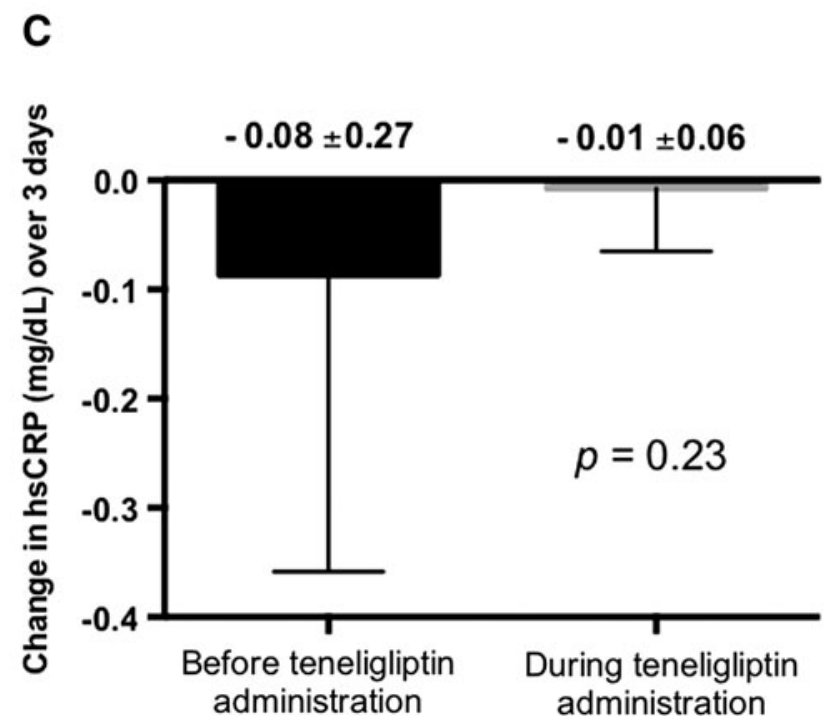

FIG. 2. Mean (SD) changes with administration of teneligliptin in indexes of blood glucose control and inflammatory parameters: (A) glycated albumin (GA), (B) 1,5-anhydro-D-glucitol (1,5-AG), and (C) high-sensitivity C-reactive protein (hsCRP).

short-term teneligliptin with insulin therapy, with or without other antidiabetes drugs.

Several studies reported that glucose fluctuations exhibited a more specific triggering effect on oxidative stress than chronic sustained hyperglycemia. ${ }^{22,23}$ Glucose variability may be an important risk factor for cardiovascular disease. Our study demonstrated that the addition of teneligliptin to insulin therapy decreased serum GA and increased serum 1,5-AG levels after only several days, providing good control of glucose excursions. Therefore, it is possible that combination therapy of teneligliptin and insulin, with or without other antidiabetes drugs, is a beneficial option for the treatment in patients with type 2 diabetes, especially in those with diabetes complications and/or with cardiovascular disease or in older patients, who are known to be at high risk of cardiovascular disease. ${ }^{24}$

Because Satoh-Asahara et al. ${ }^{25}$ demonstrated that sitagliptin, another DPP-4 inhibitor, decreased serum hsCRP levels in patients with type 2 diabetes, we measured serum hsCRP levels to confirm any anti-inflammatory effects of teneligliptin. However, we found no significant difference in serum hsCRP levels before and after treatment with teneligliptin. This lack of anti-inflammatory effect of teneligliptin may be due to the shorter treatment period ( 7 days), the small number of subjects, or the adequately controlled subjects (24-h mean glucose level of $148.8 \pm 25.7 \mathrm{mg} / \mathrm{dL}$ ), compared with the study of Satoh-Asahara et al. ${ }^{25}$ It would be helpful to know these same values for the previous study.

This study has several limitations as a pilot study. First, the number of subjects enrolled was relatively small. The number of subjects studied in some clinical previous investigations (Mori et al., ${ }^{7}$ Osonoi et al., ${ }^{26}$ and Kim et al. ${ }^{27}$ ) of glucose fluctuations affected by antidiabetes drugs using CGM was similar to that of our study. Therefore, 26 patients might be enough to verify the effects of teneligliptin on glucose fluctuation using CGM such as in our pilot study. Second, there 
was no control group. Third, we did not use a test meal. However, the daily intake of calories was calculated by patients' ideal body weight during hospitalization, and physical activity can be regarded as practically equal, as all patients carried a pedometer to meet the target of taking 5,000 steps a day under the guideline of medical staff during admission. A large-scale clinical trial should be performed to confirm our study in patients with type 2 diabetes.

\section{Conclusions}

Our study has demonstrated that add-on treatment with teneligliptin decreased the proportion of time in hyperglycemia and increased the proportion of time at normal glucose levels in a $24-\mathrm{h}$ period and significantly improved glucose fluctuations over a 24 -h period, indicated by the SD of 24-h glucose levels and the mean amplitude of glycemic excursions. In addition, levels of serum GA and 1,5-AG, which are indexes of glucose control, were also significantly improved after treatment with teneligliptin. This effect occurred rapidly after administration of teneligliptin. Add-on treatment with teneligliptin as well as other DPP-4 inhibitors might be a beneficial option for patients with type 2 diabetes receiving insulin therapy, with or without other antidiabetes drugs.

\section{Acknowledgments}

The authors thank all the study participants. We acknowledge Atsumi Kezuka for data collection.

\section{Author Disclosure Statement}

No competing financial interests exist.

\section{References}

1. Drucker DJ: The role of gut hormones in glucose homeostasis. J Clin Invest 2007;117:24-32.

2. Holst JJ: The physiology of glucagon-like peptide 1. Physiol Rev 2007;87:1409-1439.

3. Drucker DJ, Nauck MA: The incretin system: glucagonlike peptide-1 receptor agonist and dipeptidyl peptidase-4 inhibitors in type 2 diabetes. Lancet 2006;368:1696-1705.

4. Aso Y, Terasawa T, Kato K, et al.: The serum level of soluble CD26/dipeptidyl peptidase 4 increases in response to acute hyperglycemia after oral glucose load in healthy subjects: association with high-molecular weight adiponectin and hepatic enzymes. Transl Res 2013;162:309-316.

5. Xu L, Man CD, Charbonnel B, et al.: Effect of sitagliptin, a dipeptidyl peptidase-4 inhibitor, on beta-cell function in patients with type 2 diabetes: a model-based approach. Diabetes Obes Metab 2008;10:1212-1220.

6. Deacon CF: Dipeptidyl peptidase-4 inhibitors in the treatment of type 2 diabetes: a comparative review. Diabetes Obes Metab 2011;13:7-18.

7. Mori Y, Taniguchi Y, Matsuura K, et al.: Effects of sitagliptin on 24-h glycemic changes in Japanese patients with type 2 diabetes assessed using continuous glucose monitoring. Diabetes Technol Ther 2011;13:699-703.

8. Kadowaki T, Kondo K: Efficacy, safety and dose-response relationship of teneligliptin, a dipeptidyl peptidase-4 inhibitor, in Japanese patients with type 2 diabetes mellitus. Diabetes Obes Metab 2013;15:810-818.
9. Nabeno M, Akahoshi F, Kishida $\mathrm{H}$, et al.: A comparative study of the binding modes of recently launched dipeptidyl peptidase IV inhibitors in the active site. Biochem Biophys Res Commun 2013;434:191-196.

10. Kim D, Wang L, Baconi M, et al.: (2R)-4-Oxo-4-[3(trifluoromethyl)-5,6-dihydro[1,2,4] triazolo[4,3-a]pyrazin7(8H)-yl]-1-(2,4,5-trifluorophenyl)butan-2-amine: a potent, orally activate dipeptidyl peptidase IV inhibitor for the treatment of type 2 diabetes. J Med Chem 2005;48:141-151.

11. Metzler WJ, Yanchunas J, Weighlt C, et al.: Involvement of DPP-IV catalytic residues in enzyme-saxagliptin complex formation. Protein Sci 2008;17:240-250.

12. Eckhardt M, Langkopf E, Mark M, et al.: 8-(3-(R)Aminopiperidin-1-yl)-7-buut-2-ynyl-3-methyl-1-(4-methylquinazolin-2-ylmethyl)-3,7-dihydropurine-2,6-dione (BI 1356), a highly potent, selective, long-acting, and orally bioavailable DPP-4 inhibitor for the treatment of type 2 diabetes. J Med Chem 2007;50:6450-6453.

13. Zhang Z, Wallance MB, Feng J, et al.: Design and synthesis of pyrimidinone and pyridinedione inhibitors of dipeptidyl peptidase IV. J Med Chem 2011;54:510-524.

14. Villhauer EB, Brinkman JA, Naderi GB, et al.: 1-[[(3Hydroxy-1-adamantyl)amino]acetyl]-2-cyano- $(S)$-pyrrolidine: a potent, selective, and orally bioavailable dipeptidyl peptidase IV inhibitor with antihyperglycemic properties. J Med Chem 2003;46:2774-2789.

15. Katsuno T, Ikeda H, Ida K, et al.: Add-on therapy with the DPP-4 inhibitor sitagliptin improves glycemic control in insulin-treated Japanese patients with type 2 diabetes mellitus. Endocr J 2013;60:733-742.

16. Kothny W, Foley J, Kozlovski P, et al.: Improved glycemic control with vildagliptin added to insulin, with or without metformin, in patients with type 2 diabetes mellitus. Diabetes Obes Metab 2013;15:252-257.

17. Lukashevich V, Schweizer A, Foley JE, et al.: Efficacy of vildagliptin in combination with insulin in patients with type 2 diabetes and severe renal impairment. Vasc Health Risk Manag 2013;9:21-28.

18. Mori Y, Taniguchi Y, Miyazaki S, et al.: Effects of add-on treatment with sitagliptin on narrowing the range of glucose fluctuations in Japanese type 2 diabetes patients receiving insulin therapy. Diabetes Technol Ther 2013;15: 237-240.

19. Fravel MA, McDanel DL, Ross MB, et al.: Special considerations for treatment of type 2 diabetes mellitus in the elderly. Am J Health Syst Pharm 2011;68:500-509.

20. Sakamoto M, Nishimura R, Irako T, et al.: Comparison of vildagliptin twice daily vs. sitagliptin once daily using continuous glucose monitoring (CGM): crossover pilot study (J-VICTORIA study). Cardiovasc Diabetol 2012;11:92.

21. Vilsbøll T, Rosenstock J, Yki-Järvinen H, et al.: Efficacy and safety of sitagliptin when added to insulin therapy in patients with type 2 diabetes. Diabetes Obes Metab 2010;12: 167-177.

22. Monnier L, Mas E, Ginet C, et al.: Activation of oxidative stress by acute fluctuations compared with sustained chronic hyperglucemia in patinets with type 2 diabetes. JAMA 2006; 295:1681-1687.

23. Suzuki K, Olar G, Modis K, et al.: Hydrogen sulfide replacement therapy protects the vascular endothelium in hyperglycemia by preserving mitochondrial function. Proc Natl Acad Sci USA 2011;108:13829-13834.

24. Moreno G, Mangione CM: Management of cardiovascular disease risk factors in older adults with type 2 diabetes 
mellitus: 2002-2012 literature review. J Am Geriatr Soc 2013;61:2027-2037.

25. Satoh-Asahara N, Sasaki Y, Wada H, et al.: A dipeptidase-4 inhibitor, sitagliptin, exerts anti-inflammatory effects in type 2 diabetic patients. Metabolism 2013;62:347-351.

26. Osonoi T, Saito M, Tamasawa A, et al.: Effects of sitagliptin or mitiglinide as an add-on to acarbose on daily blood glucose fluctuations measured by $72 \mathrm{~h}$ subcutaneous continuous glucose monitoring in Japanese patients with type 2 diabetes: a prospective randomized study. Expert Opin Pharmacother 2014;15:1325-1335.

27. Kim HS, Shin JA, Lee SH, et al.: A comparative study of the effects of a dipeptidyl peptidase-IV inhibitor and sul- fonylurea on glucose variability in patients with type 2 diabetes with inadequate glycemic control on metformin. Diabetes Technol Ther 2013;15:810-816.

Address correspondence to: Kunihiro Suzuki, MD, PhD Department of Endocrinology and Metabolism Dokkyo Medical University 880 Kitakobayashi Mibu, Shimotsugagun, Tochigi 321-0293, Japan

E-mail: kuni-s@dokkyomed.ac.jp 